\title{
Circulating microRNA-92a level predicts acute coronary syndrome in diabetic patients with coronary heart disease
}

\author{
Wenyi Wang ${ }^{1}$, Zhigang $\mathrm{Li}^{1 *}$, Yashu Zheng ${ }^{1}$, Meiling Yan², Yameng Cui ${ }^{1}$ and Jiechun Jiang ${ }^{3}$
}

\begin{abstract}
Purpose: This study was designed to explore the value of monitoring miR-92a in T2DM patients with coronary heart disease (CHD).

Materials and methods: 40 ACS patients with prior history of CHD and diabetes while the onset time of diabetes preceded that of CHD by more than 2 years were enrolled as the DACS group(diabetic ACS group). 40 ACS subjects who had had a definite diagnosis of CHD for more than 2 years with no history of T2DM were recuited as the CACS group(chronic CHD with ACS group). All enrolled subjects from DACS and CACS group came from an emergency basis and diagnosed with ACS by coronary angiography. Another 68 age- and sex-matched volunteers with chronic stable CHD without diabetes history were assigned as the control group (CHD group). We examined the serum levels of miR-92a and analyzed their correlations with blood pressure, glucose level, and lipid level.

Results: The levels of miR-92a were significantly elevated in the DACS group compared with those of the CACS and CHD groups. Multivariate analysis showed that miR-92a, systolic blood pressure (SBP), and glycosylated hemoglobin (HbA1c) were significantly related to ACS events in patients with T2DM. Forward stepwise binary logistic regression analysis identified miR-92a as an independent predictive factor for ACS events in the patients with T2DM.

Conclusion: An elevated circulating miR-92a level was associated with an increased risk of ACS in CHD patients with T2DM. Thus the level of miR-92a, especially combined with elevated SBP and HbA1c, may be helpful in the detection of ACS in patients with T2DM.
\end{abstract}

Keywords: Acute coronary syndrome, microRNA-92a, Type 2 diabetes mellitus, Coronary heart disease

\section{Introduction}

Acute coronary syndrome (ACS) is an acute cardiac ischemic syndrome due to coronary artery atherosclerotic thrombosis involving the rupture or erosion of unstable plaques [1]. More than half of cardiovascular deaths are caused by ACS, and hyperglycemia is an independent risk factor for cardiovascular events [2]. In short, patients suffering from type 2 diabetes mellitus (T2DM) and ACS face an increased risk of adverse cardiovascular events.

Patients with coronary heart disease (CHD) and T2DM, as compared with nondiabetic patients, often have complicated vascular lesions of increasing severity

\footnotetext{
* Correspondence: 315981536@qq.com

'International Medical Center, Tianjin First Central Hospital, No. 24 of Fukang Road, Nankai District, Tianjin 300192, China

Full list of author information is available at the end of the article
}

as well as multivessel and diffuse vasculopathy [3]. Previous studies point to several circulating biomarkers related to the incidence of ACS in T2DM patients, such as glycosylated hemoglobin (HbA1c) [4], B-type natriuretic peptide (BNP) [5], phospholipid protein [6], gremlin 1, and macrophage migration inhibitory factor [7]. However, these biomarkers are of limited use because they are nonspecific. Therefore the clinical diagnosis of blood glucose-related ACS events still focuses on the typical clinical symptoms and findings of invasive imaging. Because of this, it would be useful to find specific markers that could facilitate early intervention in ACS patients with T2DM. In other words, this would provide a new approach of great socioeconomic value for the prevention and treatment of vascular complications in patients at risk. 
As small noncoding RNA molecules, the microRNAs (miRNAs) regulating gene expression at the posttranscriptional level have emerged as playing a fundamental role in many diseases. In biological research much has been achieved regarding the miRNAs, which are now known to participate in the pathogenesis of T2DM mellitus and related complications [8]. Accordingly, studies have found aberrant expression of miR-143, miR-21, miR-486-3p, miR-100, miR-92a, miR-3135b, and miR-223 in ACS patients [9-15]. In studies of ACS in T2DM (referred to here as diabetic ACS, or DACS), miR-92a attracts particular attention because it is a newly recognized biomarker of ACS [13] and hyperglycemia [16] in patients with CHD; also, it regulates neovascularization, and the inhibition of miR-92a enhances angiogenesis [17]. There is compelling evidence that miR-92a plays a role in the pathogenesis of ACS and that high levels of expression of miR-92a can serve as a potential biomarker to differentiate between patients with stable CAD and those with acute myocardial infarction (AMI). In AMI, miR-92a can even point to the activation of endothelial cells [18]. In coronary sinus samples, miR-92a-3p was found to be significantly downregulated, and left ventricular filling pressure was negatively correlated with miR-92a-3p [19]. How miRNA-92a exerts its influence on the pathogenesis of T2DM and related complications, especially ACS in CHD, is unclear. We tested the hypothesis that levels of miR-92a would have a significant positive correlation with rates of morbidity and mortality in CHD patients with T2DM and that levels of miR-92a could be assessed from samples of peripheral blood. In order to test this hypothesis, we studied both the expression pattern and clinical relevance of circulating miR-92a with T2DM in CHD and ACS patients with T2DM.

\section{Material and methods \\ Ethics}

This study was approved by the Ethics Committee of Tianjin First Center Hospital in Tianjin, China. Each participant in the study received both written and oral information concerning the goals of the study and each gave informed consent.

\section{Patients}

Inpatients with ACS from October 2014 to March 2016 were recruited in this study. According to current guidelines on ACS, eligible ACS subjects included patients with symptoms of decreased blood flow to the heart, such as chest pains, ST-segment elevation on the ECG, abnormal myocardial markers such as cardiac troponin (cTn), and/or a D-dimer. We found more than 230 patients who had been hospitalized on an emergency basis and, after coronary angiography, had been diagnosed with ACS. T2DM history, random blood glucose (RBG), HbA1c, fibrinogen (FIB), cTn, and blood lipids were recorded immediately on admission. After diabetic microangiopathy screening-including temperature sensory examination, nylon inspection, vibration examination, and electroencephalography-T2DM subjects with microvascular complications were excluded. The DACS group included those ACS patients who had had a history of T2DM for more than 2 years and the onset time of diabetes preceded that of CHD by more than 2 years. The CACS group included those ACS patients who had had a definite diagnosis of CHD for more than 2 years with no history of T2DM. Finally, 40 cases were enrolled as the DACS group and another 40 with no diabetes history as the CACS group. Another 68 age- and sex-matched volunteers with chronic stable CHD were recruited to serve as the control group ( $\mathrm{CHD}$ group). Chronic stable coronary heart disease includes stable angina pectoris diagnosed by the load ECG exercise test and coronary artery angiography.

The following conditions were excluded: (1) type 1 diabetes mellitus (T1DM), diabetic ketoacidosis, and coma; (2) malignant tumor, diseases of the hematologic system, immune system diseases; (3) acute inflammation, hemorrhage, pregnancy; (4) severe hepatic and/or renal dysfunction, psychological problems.

\section{Clinical measurements}

An Omron HEM-7136 monitor (Japan) was adopted to measure diastolic blood pressure (DBP) and systolic blood pressure (SBP). Chemiluminescence immunoassays were used to determine the plasma cTn concentration according to the protocol of the manufacturer (Beckman Coulter, Fullerton, CA). Plasma random blood glucose (RBG), high-density lipoprotein cholesterol (HDL-C), low-density lipoprotein cholesterol (LDL-C), total glyceride (TG), and total cholesterol (TC) levels were all determined by an enzymatic method using reagents from Roche Diagnostic on a Roche Automatic Biochemical Analyzer Modular DPP (Roche, Switzerland). FIB levels were determined using a coagulation method on an ACL-TOP700 instrument (Walfen, USA). HbA1c was detected by ion-exchange high-performance liquid chromatography on a Bio-Rad Variant $^{\mathrm{Tm}}$ II Hemoglobin Testing System (BIO-RAD, USA). All results were carried out as means of 3 tests.

\section{Processing of samples and RNA extraction}

Peripheral blood $(2 \mathrm{~mL})$ from each participant was placed in an heparin tube. A commercial Trizol reagent (Invitrogen, Carlsbad, CA,) was utilized to isolate the total RNA from serum according to the protocol of the manufacturer. Samples were then lysed in Trizol reagent and mixed with chloroform. The lysate was centrifuged so that protein, DNA, and RNA could be separated; the serum-Trizol homogenate wAS centrifuged at 12,000 r/ min for $10 \mathrm{~min}$ at $4{ }^{\circ} \mathrm{C}$; the supernatant was then 
transferred to microcentrifuge tubes. Total RNA was recovered with the precipitation of isopropanol and rinsed in $75 \%$ ethanol to eliminate impurities. Finally the total RNA was dissolved in RNase-free water and concentrated in a volume of $20 \mu \mathrm{L}$ with an Eppendorf Concentrator Plus 5301 (Eppendorf, Germany). A Nano Drop ND-1000 (Nano Drop, Wilmington, DE) was used to quantify the concentration of each RNA sample. All RNA samples were stored at $-80^{\circ} \mathrm{C}$ until use.

miRNA expression and real-time reverse transcriptase polymerase chain reaction (RT-PCR).

The miRNA was quantified by quantitative and real-time polymerase chain reaction (RT-PCR) from total RNA; it was polyadenylated and reversely transcribed into cDNA in a final volume of $20 \mu \mathrm{L}$ using the PrimeScript RT reagent kit (Takara, Bio Inc., Tokyo, Japan). RT-PCR was implemented in duplicate measurements by utilizing SYBR Premix Ex Taq ${ }^{\mathrm{Tm}}$ II (Takara, Bio Inc., Tokyo, Japan) in a 7300 quantitative PCR system (Applied Biosystems). The miRNA-specific primer sequences were designed on the basis of miRNA sequences that were acquired via the miRBase database (http://www.mirbase.org//) (UAUUGCACUUGUCC CGGCCUGU). All amplification reactions were made in one final volume of $20 \mu \mathrm{L}$ that contained $1 \mu \mathrm{L}$ of cDNA, $0.25 \mathrm{~mm}$ of primer, and $1 \times$ SYBR Green PCR Master mix in accordance with the cycling conditions recommended by the supplier ( 40 cycles of $30 \mathrm{~s}$ at $90^{\circ} \mathrm{C}, 5 \mathrm{~s}$ at $95^{\circ} \mathrm{C}$, and $31 \mathrm{~s}$ at $60^{\circ} \mathrm{C}$ ). Upon completion of the PCR cycles, melting curve analyses and electrophoresis of products on $3.0 \%$ agarose gels were conducted to corroborate generation of the anticipated PCR product. All samples were run in triplicate. The levels of expression of miRNAs were normalized to miR-92a, and calculated using the $2^{-\Delta \Delta \text { ct }}$ method.

\section{Statistical analysis}

SAS software, version 9.3 (SAS Institute, Cary, NC), was used to conduct statistical analyses. Continuous variables were demonstrated as averages (standard deviation) and compared by one-way analysis of variance (ANOVA) or median (interquartile range) and Kruskal-Wallis analysis according to the Shapiro-Wilk test. These analyses were followed by Bonferroni comparisons. Each probability value was two-sided and a $P$ value $<0.05$ was considered statistically significant. The correlation between miR-92a level and clinical data-including hemodynamics parameters, blood glucose parameters, and blood lipid parameters-were all evaluated by the calculating Spearman's rank correlation coefficients. The curve analysis of receiver operating characteristics (ROCs) was used to assess the diagnostic accuracy of serum biomarker levels. The optimal cutoff values for defining DACS were calculated on the basis of maximizing the sensitivity and specificity of each index. Analyses of forward stepwise binary logistic regression were conducted to determine the optimal combination of biomarkers so that DACS could be predicted.

\section{Results}

\section{Patient characteristics}

A total of 68 CHD control subjects, 40 CACS patients, and 40 DACS patients were included in this study. The participants' mean age and sex were similar.

The mean RBG was $4.86 \mathrm{mmol} / \mathrm{L}$ (interquartile range [IQR] 4.42-5.37 mmol/L) in the CHD controls, $9.02 \mathrm{mmol} /$ L (IQR 6.58-12.16 mmol/L) in the CACS group, and 11.51 $\mathrm{mmol} / \mathrm{L}$ (IQR $8.30-14.39 \mathrm{mmol} / \mathrm{L}$ ) in the DACS group. The HbAlc was $4.68 \%$ (IQR 4.47-4.94\%) in the CHD controls, $5.35 \%$ (IQR,4.78-5.86\%) in the CACS group, and $8.00 \%$ (IQR 7.27-9.60\%) in the DACS group. Both RBG and $\mathrm{HbA1c}$ were dramatically increased in the CACS and DACS groups compared with the CHD control group $(P<$ 0.001). The CACS and DACS groups had higher levels of triglyceride (TG) and total cholesterol (TC) than the CHD control group $(P<0.001)$. Both HDL-C and LDL-C were dramatically decreased in the DACS group compared with the CACS group, with $88.57 \%$ of the patients in the CACS group having normal levels of statins compared with 95.73\% in the DACS group (Table 1).

Expression of miR-92a was more clearly elevated in the DACS group than that in the CACS and CHD groups (all $P<0.017$ ). In addition, an apparent difference was also found between the CACS and CHD groups (Table 1 and Fig. 1).

\section{Expression of miR-92a}

By and large plasma levels of miR-92a were similar in male and female subjects. No correlation was found between age and the level of miR-92a after adjustment by group. However, miR-92a expression was more clearly elevated in the DACS group than in the CACS and CHD groups $(P<$ 0.001). In addition, an apparent difference was also found between CACS group and CHD group (Fig. 1, Table 1).

\section{Correlation between miR-92a level and clinical measurements}

The correlation between clinical measurements and miR-92a level was further analyzed. By utilizing Spearman's rank correlation analysis, it was demonstrated that miR-92a level was correlated with D-dimer after adjustment by group, which is excluded subsequently (Table 2 , Additional file 1 Figures S1-S3).

\section{The potential of miR-92a to predict ACS in patients with T2DM}

Both univariate and multivariate analyses were further conducted, so that factors associated with the prediction of 
Table 1 Clinical characteristics of enrolled subjects

\begin{tabular}{|c|c|c|c|c|}
\hline Variables & CHD controls $(n=68)$ & CACS $(n=40)$ & DACS $(n=40)$ & $P$ value \\
\hline Age, year & $47(44,52)$ & $52.5(49.5,57)$ & $52.5(50,57)$ & 0.964 \\
\hline Sex, male \% & $47(69.12)$ & $24(60.00)$ & $32(80.00)$ & 0.150 \\
\hline $\mathrm{BMI}, \mathrm{kg} / \mathrm{m}^{2}$ & $20.68(19.90,21.47)$ & $20.23(19.35,21.12)$ & $20.96(19.92,21.66)$ & 0.046 \\
\hline Smoking Index & $410(215,565)$ & $420(210,790)$ & $460(200,740)$ & 0.645 \\
\hline \multicolumn{5}{|l|}{ Hemodynamics } \\
\hline $\mathrm{SBP}, \mathrm{mmHg}$ & $112(102.5,119)$ & $123(117,131)^{* *}$ & $134.5(124,154.5)^{* *},+$ & $<0.001$ \\
\hline $\mathrm{DBP}, \mathrm{mmHg}$ & $74(69,81)$ & $79.5(71.5,84)$ & $80(75,90)^{*}$ & 0.003 \\
\hline \multicolumn{5}{|c|}{ Coagulation Function } \\
\hline $\mathrm{FIB}, \mathrm{g} / \mathrm{L}$ & $2.55(2.34,2.91)$ & $2.81(2.53,3.07)$ & $3.18(2.69,3.67)^{* *}$ & $<0.001$ \\
\hline D-dimer, $\mu \mathrm{g} / \mathrm{L}$ & $228.210(120.86,303.89)$ & $339.20(224.65,491.81)^{* *}$ & $333.40(265.21,417.22)$ & $<0.001$ \\
\hline \multicolumn{5}{|l|}{ Myocardial Marker } \\
\hline $\mathrm{cTn}, \mathrm{ng} / \mathrm{ml}$ & $0.03(0.02,0.04)$ & $0.02(0.01,0.03)$ & $0.05(0.01,3.07)+$ & 0.010 \\
\hline \multicolumn{5}{|l|}{ Blood Glucose } \\
\hline $\mathrm{RBG}, \mathrm{mmol} / \mathrm{L}$ & $4.86(4.42,5.37)$ & $9.02(6.58,12.16)^{* *}$ & $11.51(8.30,14.39)^{* *},+$ & $<0.001$ \\
\hline $\mathrm{HbA} 1 \mathrm{C}, \%$ & $4.68(4.47,4.94)$ & $5.35(4.78,5.86)^{* *}$ & $8.00(7.27,9.60)^{* *,},+\dagger$ & $<0.001$ \\
\hline \multicolumn{5}{|l|}{ Blood Lipids } \\
\hline $\mathrm{LDL}-\mathrm{C}, \mathrm{mmol} / \mathrm{L}$ & $2.66(2.42,2.89)$ & $3.11(2.63,3.92)^{* *}$ & $2.61(2.15,3.39) \dagger$ & $<0.001$ \\
\hline $\mathrm{TG}, \mathrm{mmol} / \mathrm{L}$ & $1.04(0.77,1.34)$ & $1.48(0.85,2.37)^{* *}$ & $1.59(0.95,3.40)^{* *}$ & $<0.001$ \\
\hline $\mathrm{TC}, \mathrm{mmol} / \mathrm{L}$ & $3.98(2.04,4.76)$ & $4.84(4.26,5.42)^{* *}$ & $4.56(3.26,6.27)^{*}$ & $<0.001$ \\
\hline $\mathrm{HDL}-\mathrm{C}, \mathrm{mmol} / \mathrm{L}$ & $0.70(0.41,1.01)$ & $1.04(0.87,1.32)^{* *}$ & $0.92(0.55,1.19)^{\dagger}$ & $<0.001$ \\
\hline \multicolumn{5}{|l|}{ Biomarkers } \\
\hline $\operatorname{miR}-92 a^{a}$ & $2.39(1.03)$ & $3.88(0.78)^{* *}$ & $5.60(1.22)^{* *,+t}$ & $<0.001$ \\
\hline Treatment & $\begin{array}{l}\text { antiplatelet therapy }(81.16 \%) \text {, } \\
\text { nitrates }(43.49 \%), \text { ACEI/ARB } \\
(75.36 \%) \text {, statins }(79.71 \%), \\
\text { beta-blockers }(40.58 \%)\end{array}$ & $\begin{array}{l}\text { antiplatelet therapy }(90.00 \%) \text {, } \\
\text { nitrates }(85.71 \%), \text { ACEI/ARB } \\
(72.86 \%), \text { statins }(88.57 \%) \text {, } \\
\text { beta-blockers }(54.29 \%) \text {, } \\
\text { diuretics }(62.86 \%)\end{array}$ & $\begin{array}{l}\text { antiplatelet therapy }(81.20 \%) \text {, nitrates } \\
(70.09 \%), \text { ACEI/ARB }(87.18 \%) \text {, statins }(95.73 \%) \text {, } \\
\text { beta-blockers }(29.91 \%) \text {, biguanides }(24.79 \%) \text {, } \\
\text { insulins }(41.88 \%)\end{array}$ & \\
\hline
\end{tabular}

\#All the continuous variables were compared using Kruskal-Wallis analysis and presented by median (interquartile range) for their non-normal distribution unless there are some additional notes such as a. One-way analysis of variance was adopted to evaluate the equality of marked indicator, which was presented by average (standard deviation). ${ }^{*} P<0.017,{ }^{* *} P<0.001$ vs. NC group; $+P<0.017,+\uparrow P<0.001$ vs. CACS group.

DACS could be identified. Univariate analysis was adopted to select predictive factors of DACS, revealed that sex, body mass index (BMI), SBP, FIB, cTn, RBG, HbA1c, LDL-C, HDL-C, and miR-92a were all closely associated with DACS. Predictive factors selected from the univariate analysis were utilized as variables for forward stepwise multivariate logistic regression. SBP, miR-92a, and HbA1C were further added to the multivariate logistic regression analysis. miR-92a (OR 95\% CI: 11.011 [1.967, 61.630], $P=0.006$ ) was confirmed as an independent predictive factor for DACS among CHD patients (Table 3).

ROC analysis determined the diagnostic accuracy of HbA1C, miR-92a, and SBP, as shown in Table 4. The miR-92a separately (cutoff $>4.65$ ) showed $85 \%$ sensitivity and $82.50 \%$ specificity with an AUC of 0.883 in the prediction of DACS. The forward stepwise binary logistic regression analysis showed that the combination of HbA1C, SBP, and miR-92a had a better predictive value, with an AUC of 0.986, than the combination of any individual biomarker taken separately.

$$
P=\frac{1}{1+\exp (-Z)}
$$

The probability of $\mathrm{HbA} 1 \mathrm{C}, \mathrm{SBP}$, and miR-92a was calculated by adopting the following logistic formula in the prediction of diabetic ACS: $Z=\beta 0+\beta 1 X 1+\beta 2 X 2+\ldots$ $+\beta \mathrm{mXm} . \beta 0$ is a constant, and $\beta 1, \beta 2 \ldots \beta \mathrm{m}$ refers to the estimated regression coefficients of risk factors. Therefore the predicted probability $(P)$ was calculated by the following formula:

$$
P=\frac{1}{1+\exp \left(47.00-0.12^{*} S B P-3.28^{*} H b A 1 C-2.40^{*} \text { miR-92a }\right)}
$$

The combination of HbA1C, elevated SBP, and miR-92a demonstrated $95 \%$ sensitivity and $95 \%$ 


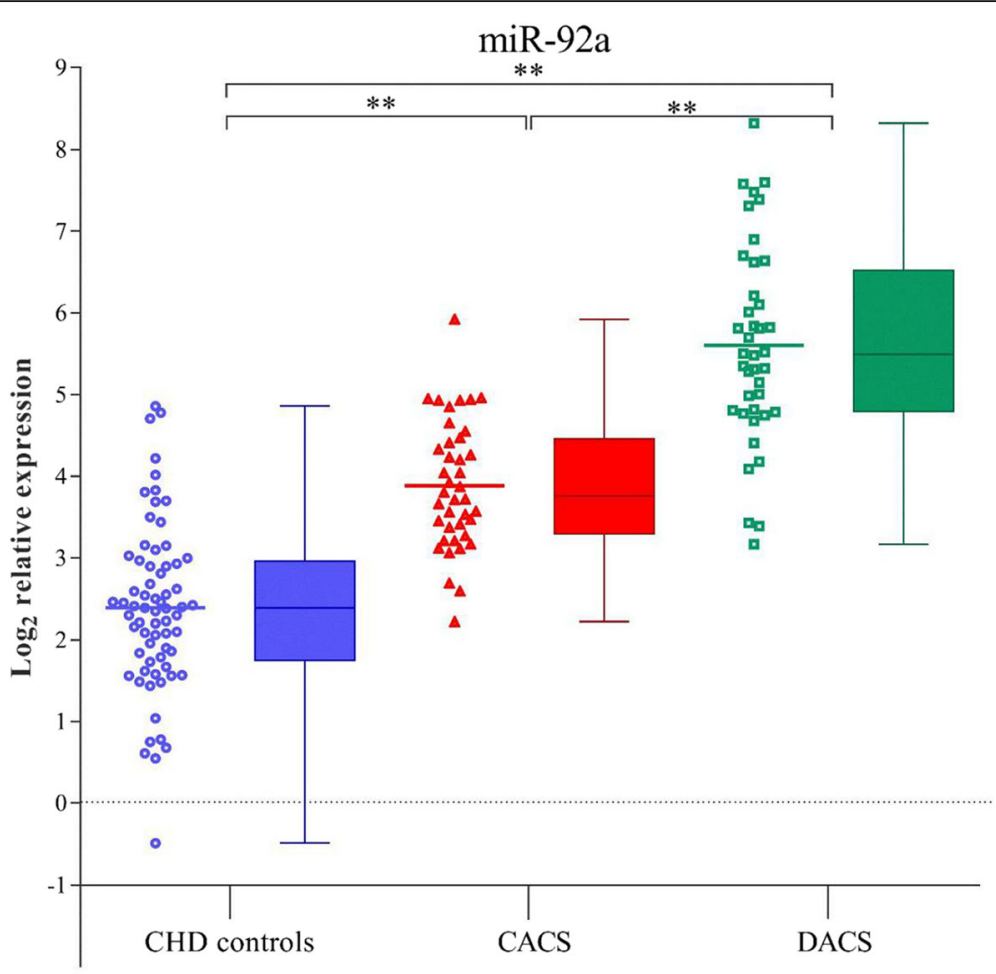

Fig. 1 Levels of miR-92a in the CHD, CACS, and DACS groups. The box-and-whiskers plot was done via Turkey method. ${ }^{* *} p<0.001$

Table 2 Spearman's correlation coefficients of miR-92a level with clinical data

\begin{tabular}{|c|c|c|c|c|}
\hline \multirow[t]{2}{*}{ Variables } & \multicolumn{2}{|c|}{ No adjusted } & \multicolumn{2}{|c|}{ Adjusted by group } \\
\hline & $r$ value & $P$ value & $r$ value & $P$ value \\
\hline Age & 0.06 & 0.592 & -0.01 & 0.913 \\
\hline Male Sex & -0.03 & 0.746 & 0.05 & 0.556 \\
\hline BMI & -0.10 & 0.228 & -0.11 & 0.188 \\
\hline Smoking Index & 0.01 & 0.948 & -0.09 & 0.303 \\
\hline \multicolumn{5}{|l|}{ Hemodynamics } \\
\hline SBP & 0.53 & $<.001$ & 0.09 & 0.280 \\
\hline $\mathrm{DBP}$ & 0.26 & 0.002 & 0.06 & 0.454 \\
\hline \multicolumn{5}{|c|}{ Coagulation function } \\
\hline FIB & 0.30 & $<.001$ & -0.01 & 0.922 \\
\hline D-dimer & 0.17 & 0.040 & -0.21 & 0.011 \\
\hline \multicolumn{5}{|c|}{ Myocardial markers } \\
\hline cTn & 0.15 & 0.070 & 0.07 & 0.379 \\
\hline \multicolumn{5}{|l|}{ Blood Glucose } \\
\hline RBG & 0.64 & $<.001$ & 0.07 & 0.368 \\
\hline $\mathrm{HbA1C}$ & 0.60 & $<.001$ & 0.01 & 0.909 \\
\hline \multicolumn{5}{|l|}{ Blood Lipids } \\
\hline LDL-C & 0.05 & 0.570 & -0.01 & 0.857 \\
\hline TG & 0.22 & 0.008 & -0.15 & 0.076 \\
\hline TC & 0.20 & 0.013 & -0.05 & 0.517 \\
\hline HDL-C & 0.19 & 0.020 & 0.06 & 0.457 \\
\hline
\end{tabular}

specificity in predicting DACS among patients with CHD (Table 4, Fig. 2, Additional file 1 Figure S4).

\section{Discussion}

The significance of circulating biomarkers for predicting DACS in CHD patients has aroused great interest. This study demonstrates that miR-92a levels rose dramatically in DACS patients and had no correlation with blood glucose or blood lipids levels. Our univariate logistic regression analysis showed that the miR-92a and SBP, FIB, cTn, RBG, HbA1c, LDL-C, and HDL-C were significantly related to the DACS event. It further demonstrated that miR-92a was an independent predictive factor for the DACS.

The overall incidence of CHD and ACS has been rising, with serious implications for public health; moreover, T2DM is an important risk factor for cardiovascular disease [20]. According to current diabetes guidelines, DACS is a macrovascular complication of T2DM [21]. Compared with the nondiabetic CHD population, the age of onset of atherosclerosis in patients with CHD and T2DM is earlier; its progression is more rapid and complicated by vascular complications, multivessel disease, and other diffuse lesions [22]. The mechanism accelerating atherosclerosis can simultaneously cause an ACS event. Among the specific mechanisms controlling DACS and the integrative function of cardiovascular diseases, noncoding miRNA sequences have drawn much attention [14]. For example, miR-92a is part of the miR-17-92 cluster of miRNAs and 
Table 3 Univariate and multivariate logistic regression analysis of variables related to diabetics ACS due to coronary hear desease

\begin{tabular}{|c|c|c|c|c|}
\hline \multirow[t]{2}{*}{ Variables } & \multicolumn{2}{|l|}{ Univariate analysis } & \multicolumn{2}{|l|}{ Multivariate analysis } \\
\hline & OR $(95 \% C l)$ & $P$ value & OR $(95 \% C I)$ & $P$ value \\
\hline Age, year & $1.001(0.919-1.09)$ & 0.983 & - & - \\
\hline Sex,male \% & $0.375(0.138-1.020)$ & 0.055 & - & - \\
\hline $\mathrm{BMl}, \mathrm{kg} / \mathrm{m}^{2}$ & $1.534(0.998-2.357)$ & 0.051 & - & \\
\hline Smoking Index & $1.000(0.999-1.001)$ & 0.835 & & \\
\hline $\mathrm{SBP}, \mathrm{mmHg}$ & $1.039(1.011-1.067)$ & 0.006 & $1.127(1.024-1.239)$ & 0.014 \\
\hline $\mathrm{DBP}, \mathrm{mmHg}$ & $1.020(0.977-1.064)$ & 0.366 & - & - \\
\hline $\mathrm{FIB}, \mathrm{g} / \mathrm{L}$ & 2.059(1.082-3.919) & 0.028 & - & - \\
\hline D-dimer & 0.999(0.996-1.002) & 0.627 & & \\
\hline cTn\#, ng/ml & $1.824(1.213-2.743)$ & 0.004 & - & - \\
\hline $\mathrm{RBG}, \mathrm{mmol} / \mathrm{L}$ & $1.181(1.043-1.336)$ & 0.009 & - & - \\
\hline $\mathrm{HbA} 1 \mathrm{C}, \%$ & $6.147(2.657-14.219)$ & $<.001$ & $25.707(2.279-313.031)$ & 0.009 \\
\hline $\mathrm{LDL}-\mathrm{C}, \mathrm{mmol} / \mathrm{L}$ & $0.492(0.286-0.846)$ & 0.010 & - & - \\
\hline $\mathrm{TG}, \mathrm{mmol} / \mathrm{L}$ & $1.100(0.887-1.365)$ & 0.385 & - & - \\
\hline $\mathrm{TC}, \mathrm{mmol} / \mathrm{L}$ & $0.870(0.650-1.164)$ & 0.347 & - & - \\
\hline $\mathrm{HDL}-\mathrm{C}, \mathrm{mmol} / \mathrm{L}$ & $0.113(0.027-0.485)$ & 0.003 & - & - \\
\hline miR-92a & $6.006(2.757-13.085)$ & $<.001$ & $11.011(1.967-61.630)$ & 0.006 \\
\hline
\end{tabular}

Note: \#The data was "transformed" by log-exponential transform before being added to the logistic regression

has been recognized as a significant proatherogenic mechano-miRNA [23]. miR-92a is expressed in endothelial cells in the aortic arch, and a study by de Winther [24] showed that it induces endothelial dysfunction and a proatherogenic response. Further research showed that miR-92a induced endothelial inflammation by targeting KLF2 and KLF4 [25] or via the NRF2-KEAP1-ARE pathway [26]' even controlling cholesterol levels and Golgi volume via protein secretion [27]. Nevertheless, there is as yet no study showing whether miR-92a plays a role in DACS.

miRNAs can be released into the circulation; they participate in regulating gene expression both intracellularly and at a distance. In our study, 80 patients with ACS were enrolled well as $68 \mathrm{CHD}$ control subjects and their plasma miR-92a levels were analyzed. It was observed that miR-92a level was comparable between male subjects and female subjects. Furthermore, miR-92a levels had no correlation with age or BMI. Nevertheless the circulating level of miR-92a was apparently different between DACS patients and those without diabetes. In particular, miR-92a levels in DACS patients increased to a greater degree than those in nondiabetic ACS patients, implying that miR-92a may play a role in the progression of disease in DACS patients.

In clinical practice, hypertension is regarded as a major risk factor for ACS among CHD patients. Other risk factors include hyperlipidemia, especially $\mathrm{TC}$ and LDL-C, hyperglycemia, smoking, obesity, and genetics. In accordance with prior reports, our study demonstrated that SBP and blood glucose were significantly increased in ACS patients other than CHD patients. Furthermore, SBP, cTn, RBG, and HbAlc were elevated more markedly in the DACS group that the CACS group, while HDL-C was decreased. The miR-92a level was also correlated with changes in HbA1c and SBP. Furthermore, the level of miR-92a was not correlated with blood glucose or lipid level. These findings show that miR-92a can predict ACS events in patients with T2DM and CHD may impair the stability of atherosclerotic plaque.

Table 4 Data from ROC curves

\begin{tabular}{llllll}
\hline Indicators & AUC $(95 \% C l)$ & $P$ value & Cut-off & Sensitivity/Specificity & PPV/NPV \\
\hline SBP & $0.687(0.573,0.786)$ & 0.002 & $>132$ & $60.00 / 77.50$ & $72.70 / 66.00$ \\
HbA1C & $0.926(0.849,0.974)$ & $<.001$ & $>6.43$ & $82.50 / 100.00$ & $100.00 / 85.10$ \\
miR-92a & $0.883(0.791,0.944)$ & $<.001$ & $>4.65$ & $85.00 / 82.50$ & $82.90 / 84.60$ \\
SBP,HbAIC,\&miR-92a ${ }^{a}$ & $0.986(0.930,0.998)$ & $<.001$ & - & $95.00 / 95.00$ & $95.00 / 95.00$ \\
\hline
\end{tabular}

Note: ${ }^{\mathrm{M} M u l t i v a r i a t e}$ logistic regression analysis is adopted to generate predicted probability; AUC refers to the area under ROC curve; $\mathrm{Cl}$ is the confidence interval; PPV represents the positive predictive value; NPV is the negative predictive value 


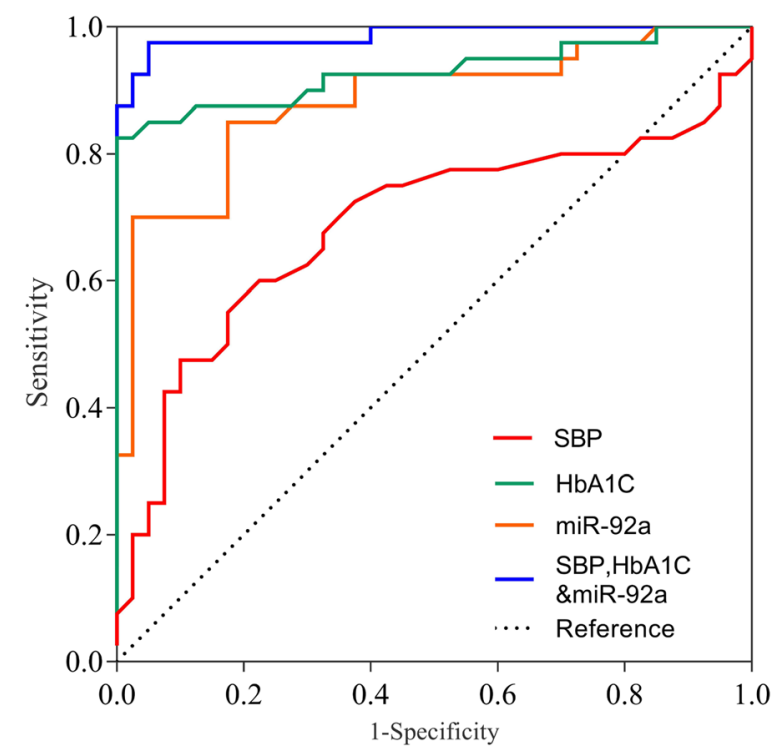

Fig. 2 ROC curve analysis. Pairwise comparison of ROC curves: $P$ (SBP vs $\mathrm{HbA1C}$ ) $<0.001 ; P$ (SBP vs miR-92a) $=0.003 ; P$ (HbA1C vs $\operatorname{miR}-92 \mathrm{a})=0.342$

Except for the traditional risk factors-which include SBP, FIB, cTn, RBG, HbA1c, LDL-C, and HDL-C-the level of miR-92a was significantly associated with the risk of DACS. Further multivariate logistic regression analyses demonstrated that HbA1c, SBP, and miR-92a were independent predictors of ACS in patients with T2DM and CHD. These results held true even after adjusting for group differences. In our study, HbA1c and the average level of blood glucose over weeks or months instead of random blood glucose (RBG) was employed in multivariate logistic regression analysis, which might be because persistent hyperglycemia is a cause of atherosclerosis [28]. Blood lipid levels were also not related to DACS. The average LDL-C of the DACS group was 2.61 $\mathrm{mmol} / \mathrm{L}$, which, according to the T2DM management algorithm published by $\mathrm{AACE} / \mathrm{ACE}$, is far below the standard of $70 \mathrm{mg} / \mathrm{dL}(1.8 \mathrm{mmol} / \mathrm{L})$ [29].

Further ROC analyses corroborated that miR-92a separately (cutoff $>4.65$ ) showed $85 \%$ sensitivity and $82.50 \%$ specificity in predicting ACS. Our forward stepwise binary logistic regression analyses proved that the combination of HbA1c, SBP, and miR-92a had a better predictive value than any individual biomarker taken separately. This combination panel demonstrated 95\% sensitivity and $95 \%$ specificity in predicting ACS among patients with T2DN and CHD. The progression of diabetes-related atherosclerosis to DACS involves complex mechanisms including hyperglycemia, abnormal lipid metabolism, hemodynamic changes, and vascular inflammatory factors [30]. Currently the possibility of using combinations of biomarkers for the prediction of
ACS among patients with T2DM and CHD is of vital importance. Our study shows that a combination of miRNA expression, systolic blood pressure, and blood sugar level may be useful in screening for and identifying ACS among such patients.

\section{Limitations}

There are several limitations to the present study. First, because it is a single-center study with a small sample size, the predictive values should be interpreted with caution. Second, is the absence of pairwise healthy subjects as a control group in whom circulating miR-92a could also be assessed. Because it is known that miRNA-92a plays a role in the pathogenesis of ACS [31], we enrolled only age- and sex-matched patients with chronic stable CHD as a negative control group. Finally, further exploration of the relationship between miR-92a and T2DM-related atherosclerosis and should be undertaken.

\section{Conclusions}

ACS is the primary cause of death in patients with CHD and T2DM. Our study suggests that an elevated level of circulating miR-92a is associated with increased ACS among patients with CHD and T2DM. In our multivariate logistic regression analysis, the level of circulating miR-92a was seen as an independent predictive factor for ACS in patients with CHD and T2DM. Moreover, miR-92a, especially miR-92a combined with HbA1c and elevated SBP, had a better predictive value for ACS in patients with CHD and T2DM. Furthermore, this study also shows that a combination of miRNA expression, elevated systolic blood pressure, and an average level of blood sugar may be helpful in screening patients and identifying those at risk for ACS.

\section{Additional file}

Additional file 1: Figure S1. Correlation of miR-92a and hemodynamics parameters. Figure S2. Correlation of miR-92a level and blood glucose. Figure S3. Correlation of miR-92a level and blood lipid. Figure S4. Data from ROC curves. (ZIP 2509 kb)

\section{Abbreviations}

ACS: Acute coronary syndrome; AMI: Acute myocardial infarction; ANOVA: One-way analysis of variance; BMI: Body mass index; BNP: B-type natriuretic peptide; BP: Blood pressure; CHD: Coronary heart disease; CTn: Cardiac troponin; DBP: Diastolic blood pressure; FIB: fibrinogen; HbA1c: Glycated hemoglobin; HDL-C: High-density lipoprotein cholesterol; IQR: Interquartile range; LDL-C: Low-density lipoprotein cholesterol; miRNA: MicroRNA; PPG: Postprandial glucose; RBG: Random blood glucose; ROCs: Receiver operating characteristics; RT-PCR: Real-time polymerase chain reaction; SBP: Systolic blood pressure; T1DM: Type 1 diabetes mellitus; T2DM: Type 2 diabetes mellitus; TC: Total cholesterol; TG: Triglyceride

\section{Acknowledgments}

We thank LetPub (http://www.letpub.com/) for its linguistic assistance during the preparation of this manuscript. 


\section{Funding}

We wish to thank Tianjin Municipal Commission of Health and Family Planning and the natural science foundation of China for supporting our work, Grant reference No.2014KY13 and 81602139 respectively.

\section{Availability of data and materials}

The datasets supporting the conclusions of this article are included within the article.

\section{Authors` information}

L., W. W., and Y. C. propose the conception and design of research; W. W., Y. Z., Y. C., and J. J. performed experiments; W. W., M. Y., and Y. C. analyzed data; W. W., M. Y., Y. C., and J. J. interpreted results of experiments; W. W. prepared figures; W. W. drafted the manuscript; Z. L., and W. W. edited and revised the manuscript; Z. L., W. W., Y. Z., M. Y., Y. C., and J. J. approved final version of the manuscript.

\section{Ethics approval and consent to participate}

The study protocol was approved by the Ethic Committee of the Tianjin First Center Hospital and conducted in accordance with Helsinki's Declaration. All the patients gave their written information consent.

\section{Consent for publication}

Not applicable.

\section{Competing interests}

The authors declare that they have no competing interests.

\section{Publisher's Note}

Springer Nature remains neutral with regard to jurisdictional claims in published maps and institutional affiliations.

\section{Author details}

'International Medical Center, Tianjin First Central Hospital, No. 24 of Fukang Road, Nankai District, Tianjin 300192, China. ${ }^{2}$ Pharmacy Department, Tianjin First Central Hospital, Tianjin, China. ${ }^{3}$ Medical Laboratory, Tianjin First Central Hospital, Tianjin, China.

Received: 23 June 2018 Accepted: 6 January 2019

Published online: 22 January 2019

\section{References}

1. Eisen A, Giugliano RP, Braunwald E. Updates on Acute Coronary Syndrome: A Review. JAMA Cardiol. 2016:1:718-30.

2. Mikhail K. Hyperglycemia in acute coronary syndromes: from mechanisms to prognostic implications. Endocrinol Metab Clin N Am. 2018:47:185-202.

3. Marso SP, Mercado N, Maehara A, Weisz G, Mintz GS, McPherson J, et al. Plaque composition and clinical outcomes in acute coronary syndrome patients with metabolic syndrome or diabetes. JACC Cardiovasc Imaging. 2012:5:\$42-52

4. McCune C, Maynard S, McClements B, Lindsay JR. HbA1c for diabetes screening in acute coronary syndrome: time for a reappraisal of the guidelines? Ulster Med J. 2015;84:154-6.

5. Wolsk E, Claggett B, Diaz R, Dickstein K, Gerstein HC, Køber L, et al. Increases in natriuretic peptides precede heart failure hospitalization in patients with a recent coronary event and type 2 diabetes mellitus. Circulation. 2017;136: 1560-2.

6. Cavusoglu E, Marmur JD, Chhabra S, Hojjati MR, Yanamadala S, Chopra V, et al. Elevated baseline plasma phospholipid protein (PLTP) levels are an independent predictor of long-term all-cause mortality in patients with diabetes mellitus and known or suspected coronary artery disease. Atherosclerosis. 2015;239:503-8.

7. Müller KA, Rath D, Schmid M, Schoenleber H, Gawaz M, Geisler T, et al. High plasma levels of Gremlin-1 and macrophage migration inhibitory factor, but not their ratio, indicate an increased risk for acute coronary syndrome in patients with type 2 diabetes mellitus. Clin Cardiol. 2016:39:201-6.

8. Liang $Y$. Jiajianghui L. Xiao H., He Y, Zhang L, Yan YX. Identification of stress-related microRNA biomarkers in type 2 diabetes: a systematic review and meta-analysis. J Diabetes 2018;Jan 17.

9. Li B, Fan J, Chen N. A novel regulator of type II diabetes: MicroRNA-143. Trends Endocrinol Metab. 2018;29:380-8.
10. Darabi F, Aghaei M, Movahedian A, Elahifar A, Pourmoghadas A Sarrafzadegan N. Association of serum microRNA-21 levels with Visfatin, inflammation, and acute coronary syndromes. Heart Vessel. 2017;32:549-57.

11. Wei T, Folkersen L, Ehrenborg E, Gabrielsen A. MicroRNA 486-3P as a stability marker in acute coronary syndrome. Biosci Rep. 2016;36:e00351.

12. Izawa H, Amano T. Plasma microRNA-100 as a biomarker of coronary plaque vulnerability - a new generation of biomarker for developing acute coronary syndrome. Circ J. 2015;79:303-4.

13. Zhang Y, Cheng J, Chen F, Wu C, Zhang J, Ren X, et al. Circulating endothelial microparticles and miR-92a in acute myocardial infarction. Biosci Rep 2017:37: BSR20170047.

14. Wang A, Kwee LC, Grass E, Neely ML, Gregory SG, Fox KAA, et al. Whole blood sequencing reveals circulating microRNA associations with high-risk traits in non-ST-segment elevation acute coronary syndrome. Atherosclerosis. 2017;261:19-25.

15. Chyrchel B, Totoń-Żurańska J, Kruszelnicka O, Chyrchel M, Mielecki W, Kołton-Wróż M, et al. Association of plasma miR-223 and platelet reactivity in patients with coronary artery disease on dual antiplatelet therapy: A preliminary report. Platelets. 2015;26:593-7.

16. Simionescu N, Niculescu LS, Carnuta MG, Sanda GM, Stancu CS, Popescu AC, et al. Hyperglycemia determines increased specific MicroRNAs levels in sera and HDL of acute coronary syndrome patients and stimulates MicroRNAs production in human macrophages. PLoS One. 2016;11: e0161201

17. Murata K, Ito H, Yoshitomi H, Yamamoto K, Fukuda A, Yoshikawa J, et al. Inhibition of miR-92a enhances fracture healing via promoting angiogenesis in a model of stabilized fracture in young mice. J Bone Miner Res. 2014;29: $316-26$

18. Loyer X, Potteaux S, Vion AC, Guérin CL, Boulkroun S, Rautou PE, et al. Inhibition of microRNA-92a prevents endothelial dysfunction and atherosclerosis in mice. Circ Res. 2014:114:434-43.

19. Ye Y, Perez-Polo JR, Qian J, Birnbaum Y. The role of microRNA in modulating myocardial ischemia-reperfusion injury. Physiol Genomics. 2010:43:534-42.

20. Newman JD, Schwartzbard AZ, Weintraub HS, Goldberg IJ, Berger JS. Primary prevention of cardiovascular disease in diabetes mellitus. J Am Coll Cardiol. 2017;70:883-93.

21. Fox CS, Golden SH, Anderson C, Bray GA, Burke LE, de Boer IH, et al. Update on prevention of cardiovascular disease in adults with type 2 diabetes mellitus in light of recent evidence: A scientific statement from the American Heart Association and the American Diabetes Association. Circulation. 2015;132:691-718.

22. Rodriguez V, Weiss MC, Weintraub H, Goldberg IJ, Schwartzbard A. Cardiovascular disease leads to new algorithm for diabetes treatment. J Clin Lipidol. 2017:11:1126-33.

23. Giral H, Kratzer A, Landmesser U. MicroRNAs in lipid metabolism and atherosclerosis. Best Pract Res Clin Endocrinol Metab. 2016:30:665-76.

24. de Winther MPJ, Lutgens E. MiR-92a: at the heart of lipid-driven endothelial dysfunction. Circ Res. 2014;114:399-401.

25. Liu H, Li G, Zhao W, Hu Y. Inhibition of mir-92a may protect endothelial cells after acute myocardial infarction in rats: role of klf2/4. Med Sci Monit. 2016;22:2451-62.

26. Liu H, Wu HY, Wang WY, Zhao ZL, Liu XY, Wang LY. Regulation of miR-92a on vascular endothelial aging via mediating Nrf2-KEAP1-ARE signal pathway. Eur Rev Med Parmacol Sci. 2017;21:2734-42.

27. Loh WP, Yang Y, Lam KP. miR-92a enhances recombinant protein productivity in $\mathrm{CHO}$ cells by increasing intracellular cholesterol levels. Biotechnol J. 2017. https://doi.org/10.1002/biot.201600488.

28. Monnier L, Colette C, Owens D. The glycemic triumvirate and diabetic complications: is the whole greater than the sum of its component parts? Diabetes Res Clin Pract. 2012;95:303-11.

29. Garber AJ, Abrahamson MJ, Barzilay Jl, Blonde L, Bloomgarden ZT, Bush MA, et al. Consensus statement by the American Association of Clinical Endocrinologists and American College of endocrinology on the comprehensive type 2 diabetes management algorithm-2016 executive summary. Endocr Pract. 2016;22:84-113.

30. Alexandru N, Badila E, Weiss E, Cochior D, Stępień E, Georgescu A. Vascular complications in diabetes: microparticles and microparticle associated microRNAs as active players. Biochem Bioph Res Commun. 2016:472:1-10.

31. Ren J, Zhang J, Xu N, Han G, Geng Q, Song J, Li S, Zhao J, Chen H. Signature of circulating microRNAs as potential biomarkers in vulnerable coronary artery disease. PloS One. 2013, e80738;8. 